\title{
ON DERIVATION OF EULER-LAGRANGE EQUATIONS FOR INCOMPRESSIBLE ENERGY-MINIMIZERS
}

\author{
NIRMALENDU CHAUDHURI AND ARAM L. KARAKHANYAN
}

\begin{abstract}
We prove that any distribution $q$ satisfying the equation $\nabla q=\operatorname{div} \mathbf{f}$ for some tensor $\mathbf{f}=\left(f_{j}^{i}\right), f_{j}^{i} \in h^{r}(U)(1 \leq r<\infty)$ -the local Hardy space, $q$ is in $h^{r}$, and is locally represented by the sum of singular integrals of $f_{j}^{i}$ with Calderón-Zygmund kernel. As a consequence, we prove the existence and the local representation of the hydrostatic pressure $p$ (modulo constant) associated with incompressible elastic energy-minimizing deformation $\mathbf{u}$ satisfying $|\nabla \mathbf{u}|^{2},|\operatorname{cof} \nabla \mathbf{u}|^{2} \in h^{1}$. We also derive the system of EulerLagrange equations for incompressible local minimizers $\mathbf{u}$ that are in the space $K_{\text {loc }}^{1,3}$ (defined in (1.2)); partially resolving a long standing problem. For Hölder continuous pressure $p$, we obtain partial regularity of area-preserving minimizers.
\end{abstract}

\section{INTRODUCTION}

Let $\Omega \subset \mathbb{R}^{n}, n \geq 2$ be a bounded Lipschitz material body. For MooneyRivlin or Neo-Hookean materials [Ba 77, [TO 81, Og 84, such as vulcanized rubber, in the equilibrium state, one is interested in minimizing the elastic energy

$$
E[\mathbf{w}]:=\int_{\Omega} L(\nabla \mathbf{w}(x)) d x,
$$

for incompressible $W^{1,2}$-deformations $\mathbf{w}: \Omega \subset \mathbb{R}^{n} \rightarrow \mathbb{R}^{n}$, subject to its own boundary condition, and corresponding to a given smooth bulk energy $L: \mathbb{M}^{n \times n} \rightarrow \mathbb{R}$. Let us define the subspace $K^{1, r}$ for $1 \leq r<\infty$, by

$$
K^{1, r}\left(\Omega, \mathbb{R}^{n}\right):=\left\{\mathbf{w} \in W^{1, r}\left(\Omega, \mathbb{R}^{n}\right): \operatorname{cof} \nabla \mathbf{w} \in L^{r}\left(\Omega, \mathbb{M}^{n \times n}\right)\right\}
$$

Date: July 24, 2008.

2000 Mathematics Subject Classification. Primary 35J60, 42A40, 73C50, 73V25.

Key words: Calderón-Zygmund kernel, elliptic equations, energy-minimizers, Euler-Lagrange equations, Hardy spaces, Newtonian potential, volume-preserving maps. 
where $W^{1, r}$ denotes the usual Sobolev spaces (see for example, GT 97, Chapter 7]) and cof $P$ is the cofactor matrix, whose $i j$-th entry is $(-1)^{i+j}$ times the determinant of $(n-1) \times(n-1)$ submatrix obtained by deleting the $i$-th row and the $j$-th column from the $n \times n$ matrix $P$. Using the identity $P^{t} \operatorname{cof} P=I d_{n} \operatorname{det} P$, it follows that $\operatorname{det} \nabla \mathbf{w} \in L^{1}$ for any $\mathbf{w} \in K^{1,2}$. Since $|P|=|\operatorname{cof} P|$ for any $P \in \mathbb{M}^{2 \times 2}$, the function spaces $K^{1, r}$ and $W^{1, r}$ are equal in $\mathbb{R}^{2}$. Let us denote the admissible set of deformations

$$
\mathcal{A}:=\left\{\mathbf{w} \in K^{1,2}\left(\Omega, \mathbb{R}^{n}\right): \operatorname{det} \nabla \mathbf{w}=1 \text { a.e. in } \Omega\right\},
$$

We call $\mathbf{u} \in \mathcal{A}$ to be a local minimizer of $E[\cdot]$ if and only if

$$
E[\mathbf{u}] \leq E[\mathbf{w}] \quad \text { for all } \mathbf{w} \in \mathcal{A} \text { and } \operatorname{supp}(\mathbf{w}-\mathbf{u}) \subset \Omega .
$$

Under the hypothesis that the energy density $L$ is smooth, polyconvex (convex function of minors) [Ba 77] and satisfies the growth condition

$$
C_{1}\left(|X|^{2}+|\operatorname{cof} X|^{2}\right)-C_{2} \leq L(X) \leq C_{3}\left(1+|X|^{2}+|\operatorname{cof} X|^{2}\right),
$$

for all $X \in \mathbb{M}^{n \times n}$, for some $C_{1}>0, C_{2} \geq 0, C_{3}>0$, where $|X|^{2}:=$ trace $\left(X^{t} X\right)$, using direct methods in the calculus of variations together with weak continuity of the determinant, J. Ball [Ba 77] proved the existence of local minimizers $\mathbf{u} \in \mathcal{A}$ of the energy $E[\cdot]$. An example of polyconvex $L$ satisfying the growth condition (1.5) is the stored-energy for incompressible isotropic Mooney-Rivlin materials in $\mathbb{R}^{3}$, given by

$$
L(X)=\frac{\mu_{1}}{2}\left(I_{1}(X)-3\right)+\frac{\mu_{2}}{2}\left(I_{2}(X)-3\right),
$$

where $I_{1}(X):=\operatorname{trace}(C)=|X|^{2}, I_{2}(X):=\frac{1}{2}\left[(\operatorname{trace}(C))^{2}-\operatorname{trace}\left(C^{2}\right)\right]=$ $|\operatorname{cof} X|^{2}$, are the first two principle invariants of the right Cauchy-Green strain tensor $C:=X^{t} X$ and $\mu_{1}, \mu_{2}$ are positive material constants.

Though the existence of the local minimizers of $E[\cdot]$ in $\mathcal{A}$ is known for over 30 years, the existence of integrable hydrostatic pressure associated with such minimizers, the derivation of system of Euler-Lagrange equations, and the partial regularity for such minimizers remains a challenging open problem. In this article we prove the following results:

(I) The $h^{r}(1 \leq r<\infty)$-integrability and local representation of any distribution $q$ satisfying the equation $\nabla q=\mathbf{f}$, where $\mathbf{f}:=\left(f_{j}^{i}\right), f_{j}^{i} \in h^{r}$, the local $r$-Hardy spaces. (Theorem 2.2 )

(II) The existence of a pressure $p \in L_{\text {loc }}^{r}$ if the minimizer is $\mathbf{u} \in$ $K_{\text {loc }}^{1,2 r}$ for some $r>1$. (Theorem 3.1) 
(III) The existence of a pressure $p \in h^{1}$ if the minimizer $\mathbf{u}$ satisfies the conditions $|\nabla \mathbf{u}|^{2},|\operatorname{cof} \nabla \mathbf{u}|^{2} \in h^{1}$. (Theorem 3.1)

(IV) The validity of the Euler-Lagrange equations if the minimizer is $\mathbf{u} \in K_{\text {loc }}^{1,3}$. (Theorem 4.1). The pair $(\mathbf{u}, p)$ satisfies the system $\operatorname{div}[D L(\nabla \mathbf{u}(x))-p(x) \operatorname{cof}(\nabla \mathbf{u}(x))]=0 \quad$ in $\Omega$,

where the divergence is taken in each rows.

(V) The partial regularity of $W^{1,3}$ area-preserving minimizers $\mathbf{u}$ for which the hydrostatic pressure $p$ is Hölder continuous with exponent $0<\alpha<1$. (Theorem 5.1)

The $L^{2}$-version of the result in $(\mathbf{I})$ is classical (see, Te 01, Remark 1.4, p 11] ), and plays an important role in incompressible fluids [Te 01]. The result in (I) is a crucial ingredient in proving (II) \& (III). The $h^{1}$ version of $(\mathbf{I})$ is quite delicate and to the best of our knowledge, it is new and may be of independent interest. For the case $r>1$, it follows that $\nabla q \in W^{-1, r}$, and adapting the classical functional-analytic approach demonstrated for $r=2$ (see [Te 01], [TO 81]), or arguing directly by duality, and solving the equation of the type

$$
\operatorname{div} \mathbf{w}=f \quad \text { in } V \subset \subset U, \mathbf{w}=0 \quad \text { in } \partial V,
$$

[Ev 98, p. 472-474], one can prove that $q \in L_{\text {loc }}^{r}$ However, both of these approaches fail to give informations for the critical case $r=1$ and does not give a representation of $q$. Whereas, our unified singular integral approach is self-contained, simple and provides the local $h^{r}$-estimate, as well as the local representation of $q$. The main ideas in our proof is to represent the localized-mollified distribution $q$ in terms of the Newtonian potential in $\mathbb{R}^{n}$ and finding its uniform bound in $h^{r}$, by using Calderón-Zygmund estimate [FS 72], CZ 52]. Finally we show that the local representation of $q$ consists the sum of Calderón-Zygmund type singular integrals of the tensor $\mathbf{f}$ (see equation (2.27) in Section 4).

For the case $n=2$, under the stronger hypothesis that the local minimizers of $E[\cdot]$ are classical ( $C^{1, \alpha}$-diffeomorphism), namely in the Sobolev space $W^{2, r}$ for some $r>2$, LeTallec and Oden [TO 81] established the system of equations in (1.7). For $n=2$, Bauman, Owen and Phillips BOP 92 proved that if a minimizer is in $W^{2, r}$ for some $r>2$, then it is smooth. For such $W^{2, r}, r>2$ minimizers, the authors in [BOP 92] argued directly on the level of the Euler-Lagrange equations exploring the existence of integrable hydrostatic pressure. Evans and Gariepy [EG 99] 
proved that any non-degenerate, Lipschitz area-preserving local minimizers of $E[\cdot]$ are $C^{1, \alpha}\left(\Omega_{0}\right)$, for some $0<\alpha<1$ for a dense open subset $\Omega_{0} \subset \Omega$. We believe that the Euler-Lagrange equations (1.7) that we derived for $K^{1,3}$-minimizers may be useful in understanding the partial regularity of such minimizers, as evidenced by the result in $(\mathbf{V})$.

In order to prove the existence of an integrable pressure $p$ associated with the local minimizer $\mathbf{u}$, we only require the additional mild assumption $|\nabla \mathbf{u}|^{2} \log \left(2+|\nabla u|^{2}\right),|\operatorname{cof} \nabla \mathbf{u}|^{2} \log \left(2+|\operatorname{cof} \nabla \mathbf{u}|^{2}\right) \in L_{\text {loc }}^{1}$. For $n=2$, to derive the system of equilibrium equations (1.7) for $(\mathbf{u}, p)$ in $\Omega$, we need $\mathbf{u}$ to be in $W^{1,3}$, whereas the best-known previous result in this direction were for $W^{2, r}$-minimizers for some $r>2$.

We organize the paper as follows. In Section 2 we prove (I); in Section 3 we prove (II) \& (III); in Section 4 we prove (IV), and finally in Section 5 we prove $(\mathbf{V})$. Throughout this article $C$ is a generic absolute constant depending on $n, U, \Omega, \mathbf{u}(\Omega), V \subset \subset \mathbf{u}(\Omega), r$, and $L$. Its value can vary from line to line, but each line is valid with $C$ being a pure positive number.

\section{Local integrability of SOlutions $\nabla q=\operatorname{div} \mathbf{f}$}

We recall some of the basic definitions and terminologies of Hardy spaces. Let $1 \leq r<\infty$. A distribution $f$ belongs to $H^{r}\left(\mathbb{R}^{n}\right)$ if and only if $f \in L^{r}\left(\mathbb{R}^{n}\right)$ and $R_{j}(f) \in L^{r}\left(\mathbb{R}^{n}\right)$ (see for example, St 93, Proposition 3 , p. 123]) for $j=1, \cdots, n$, where $R_{j}$ is the Riesz transform of $f$ given by

$$
R_{j}(f)(x):=\lim _{\varepsilon \rightarrow 0} c_{n} \int_{|y| \geq \varepsilon} \frac{y_{j}}{|y|^{n+1}} f(x-y) d y, c_{n}:=\frac{\Gamma\left(\frac{n+1}{2}\right)}{\pi^{(n+1) / 2}},
$$

so that $\widehat{R_{j}(f)}(\xi)=i \frac{\xi_{j}}{|\xi|} \widehat{f}$. In short, we will write $H^{r}\left(\mathbb{R}^{n}\right)$ as simply $H^{r}$. For $f \in H^{r}$, the norm is defined as

$$
\|f\|_{H^{r}}:=\|f\|_{L^{r}}+\sum_{j=1}^{n}\left\|R_{j}(f)\right\|_{L^{r}} .
$$

A standard result [St 70, p. 237] states that a positive function $f$, the Riesz transform $R_{j} f \in L_{\mathrm{loc}}^{1}$ if and only if $f \log (2+f) \in L_{\mathrm{loc}}^{1}$. For $1<$ $r<\infty$, a classical result asserts that $f \in H^{r}$ if and only if $f \in L^{r}$, see [St 70, p. 220]. The celebrated Fefferman duality theorem [Fe 71], [FS 72, Theorem 2], SSt 93, Theorem 1, p. 142] asserts that the dual of $H^{1}$ is the $\mathrm{BMO}$, the functions of bounded mean oscillations. The following 
theorem is due to Calderón-Zygmond CZ 52, Stein St 70, Theorem 3, p. 39], and Stein-Fefferman [FS 72, Corollary 1, p. 149-151].

Theorem 2.1 (Calderón-Zygmond, Fefferman-Stein). Let $1 \leq r<$ $\infty$ and $f \in H^{r}$. Let $G$ be a $C^{1}$ function on $\mathbb{R}^{n} \backslash\{0\}$ homogeneous of degree 0 with mean value 0 over the unit sphere $\mathbb{S}^{n-1}$, that is

$$
\int_{\mathbb{S}^{n-1}} G(x) d \sigma(x)=0
$$

Then the function defined as

$$
T_{0} f(x):=\lim _{\delta \rightarrow 0} \int_{|y| \geq \delta} \frac{G(y)}{|y|^{n}} f(x-y) d y
$$

exists a.e. and furthermore,

$$
\left\|T_{0} f\right\|_{H^{r}} \leq C_{n, r}\|f\|_{H^{r}}
$$

In particular, $R_{j}$ 's are bounded linear operator on $H^{r}$, for any $1 \leq$ $r<\infty$. Let us recall the definition of local Hardy spaces introduced by Goldberg Go 79. A distribution $f$ on $\mathbb{R}^{n}$ is said to be in the local $r$-Hardy space, written as $f \in h^{r}$, if and only if the maximal function

$$
\mathcal{M}_{\text {loc }} f(x):=\sup _{0<\varepsilon<1}\left|\left(\rho_{\varepsilon} * f\right)(x)\right|
$$

is in $L^{r}$, where $\rho_{\varepsilon}:=\varepsilon^{-n} \rho(x / \varepsilon)$, is a standard approximation of the identity. The $h^{r}$ norm of $f$ is defined to be the $L^{r}$ norm of the maximal function $\mathcal{M}_{\text {loc }} f$. It follows that if $f \in h^{r}$ then $\eta f \in h^{r}$ for any smooth cut-off function and $H^{r} \subset h^{r}$. For bounded Lipschitz domain $\Omega \subset \mathbb{R}^{n}$, we adopt the definition of Hardy spaces $h^{r}(\Omega)$ introduced by Miyachi [Mi 90]. A distribution $f$ on $\Omega$ is said to be in $h^{r}(\Omega)$ if $f$ is the restriction to $\Omega$ of a distribution $F$ in $h^{r}\left(\mathbb{R}^{n}\right)$, i.e.,

$$
\begin{aligned}
h^{r}(\Omega) & :=\left\{f \in \mathcal{D}^{\prime}(\Omega): \exists F \in h^{r}\left(\mathbb{R}^{n}\right), \text { such that }\left.F\right|_{\Omega}=f\right\} \\
& =h^{r}\left(\mathbb{R}^{n}\right) /\left\{F \in h^{r}\left(\mathbb{R}^{n}\right): F=0 \text { on } \Omega\right\} .
\end{aligned}
$$

The norm on this space is the quotient norm: the infimum of $h^{r}$ norms of all possible extensions of $f$ in $\mathbb{R}^{n}$. For $1<r<\infty$ the spaces $h^{r}(\Omega)$ is equivalent to $L^{r}(\Omega)$. For smooth bounded domains $\Omega$, the Theorem 2.1 is valid for $f \in h^{1}(\Omega)$, see [Mi 90], CKS 93. 
Theorem 2.2. Let $U \subset \mathbb{R}^{n}, n \geq 2$ be a bounded Lipschitz domain and $1 \leq r<\infty$. Let $\boldsymbol{f}=\left(f_{j}^{i}\right)$ such that $f_{j}^{i} \in h^{r}(U)$, for $1 \leq i, j \leq n$. Then the distribution $q: C_{0}^{\infty}(U) \rightarrow \mathbb{R}$ defined by

$$
\nabla q=\operatorname{div} \boldsymbol{f} \Longleftrightarrow\langle\nabla q, \boldsymbol{v}\rangle=-\int_{U} \boldsymbol{f}(x): \nabla \boldsymbol{v}(x) d x
$$

for $\boldsymbol{v} \in C_{0}^{\infty}\left(U, \mathbb{R}^{n}\right)$, is in $h^{r}(V)$, for any $V \subset \subset U$ where $A: B:=$ trace $\left(A^{t} B\right)=\sum_{i j} a_{j}^{i} b_{j}^{i}$, for $A, B \in \mathbb{M}^{n \times n}$. Furthermore, $q$ is locally represented by sum of singular integrals of $f_{j}^{i}$ (see equation (2.27), and for any $V \subset \subset U$, there exists $C>0$, depending only on $U, V$ and $r$ such that

$$
\|q\|_{h^{r}(V)} \leq C\|\boldsymbol{f}\|_{h^{r}(V)}
$$

Proof of Theorem 2.2, Let $U \subset \mathbb{R}^{n}, n \geq 2$ be a Lipschitz domain. Let $\mathbf{f}:=\left(f_{j}^{i}\right) \in \mathbb{M}^{n \times n}$ and $f_{j}^{i} \in h^{r}(U)$, for $1 \leq r<\infty$ and $1 \leq i, j \leq n$. Let $q \in \mathcal{D}^{\prime}(U)$, such that

$$
\nabla q=\operatorname{div} \mathbf{f} \text { in } \mathcal{D}^{\prime}(U) .
$$

Our idea is to mollify the equations in (2.5) and obtain uniform bound for the mollified $q$, by using Calderón-Zygmund estimate. Let $V \subset \subset U$ be a sub-domain and $0<\varepsilon<\operatorname{dist}(V, \partial U)$. Let $\rho_{\varepsilon}$ be the usual mollification kernel, and define convolution $q_{\varepsilon}: V \rightarrow \mathbb{R}$ by

$q_{\varepsilon}(x)=\left(q * \rho_{\varepsilon}\right)(x):=\left\langle q,\left(\rho_{\varepsilon}\right)_{x}\right\rangle \quad$ for $x \in V, \quad$ where $\left(\rho_{\varepsilon}\right)_{x}(y):=\rho_{\varepsilon}(y-x), \quad y \in U$

Then by the standard properties of the mollification DL 88, Proposition $1, \mathrm{p} 492], q_{\varepsilon}$ is smooth and for any $1 \leq i \leq n$

$$
\frac{\partial}{\partial x_{i}}\left(q * \rho_{\varepsilon}\right)=\frac{\partial q}{\partial x_{i}} * \rho_{\varepsilon}=q * \frac{\partial \rho_{\varepsilon}}{\partial x_{i}} .
$$

Hence mollifying the system of equations in (2.5), we obtain

$$
\nabla q_{\varepsilon}=\operatorname{div} \mathbf{f}_{\varepsilon} \quad \text { in } V,
$$

where the divergence is taken in each rows of $\mathbf{f}_{\varepsilon}:=\left(\left(f_{j}^{i}\right)_{\varepsilon}\right)$, and $\left(f_{j}^{i}\right)_{\varepsilon}:=$ $f_{j}^{i} * \rho_{\varepsilon}$ is the mollification of $\mathbf{f}$. Since $f_{j}^{i} \in h^{r}(U)$, we conclude that

$$
\left(f_{j}^{i}\right)_{\varepsilon} \rightarrow f_{j}^{i} \quad \text { strongly in } h^{r}(V) \text { as } \varepsilon \rightarrow 0,
$$

for all $1 \leq i, j \leq n$. Applying the divergence operator to the both sides of the above equation, we obtain

$$
\Delta q_{\varepsilon}=\operatorname{div}\left(\operatorname{div} \mathbf{f}_{\varepsilon}\right) \text { in } V .
$$


Since there is no control on the boundary values, we need to localize the equation (2.8). Let $W \subset \subset V \subset \subset U$. Let $\eta \in C_{0}^{\infty}\left(\mathbb{R}^{n}\right), 0 \leq \eta \leq 1$ be a cut-off function such that $\eta \equiv 1$ in $W$ and $\eta \equiv 0$ outside $V$. Let $\bar{q}_{\varepsilon}:=\eta q_{\varepsilon}$ be the localization of $q_{\varepsilon}$. Then $\bar{q}_{\varepsilon}$ is the solution of Poisson equation

$$
\Delta \bar{q}_{\varepsilon}=\bar{f}_{\varepsilon} \quad \text { in } \mathbb{R}^{n}
$$

where

$$
\begin{aligned}
\bar{f}_{\varepsilon} & :=\eta \Delta q_{\varepsilon}+2\left\langle\nabla q_{\varepsilon}, \nabla \eta\right\rangle+q_{\varepsilon} \Delta \eta \\
& =\eta \operatorname{div}\left(\operatorname{div} \mathbf{f}_{\varepsilon}\right)+2\left\langle\operatorname{div} \mathbf{f}_{\varepsilon}, \nabla \eta\right\rangle+q_{\varepsilon} \Delta \eta .
\end{aligned}
$$

Therefore $\bar{q}_{\varepsilon}$ is represented by the Newtonian potential of in $\mathbb{R}^{n}$. In other words,

$$
\bar{q}_{\varepsilon}(x)=-\int_{\mathbb{R}^{n}} \Phi(x-y) \bar{f}_{\varepsilon}(y) d y,
$$

where $\Phi$ is fundamental solution of the Laplace equation in $\mathbb{R}^{n}$ and is given by

$$
\Phi(x):= \begin{cases}-\frac{1}{2 \pi} \log |x| & \text { if } n=2 \\ \frac{1}{n(n-2) \alpha(n)} \frac{1}{|x|^{n-2}} & \text { if } n \geq 3,\end{cases}
$$

for $x \in \mathbb{R}^{n} \backslash\{0\}$, and $\alpha(n):=\frac{\pi^{n / 2}}{\Gamma\left(\frac{n}{2}+1\right)}$ is the volume of the unit ball in $\mathbb{R}^{n}$. Using (2.10) in (2.11), we obtain

$$
\begin{aligned}
\bar{q}_{\varepsilon}(x)= & -\int_{\mathbb{R}^{n}} \eta(y) \Phi(x-y) \operatorname{div}\left(\operatorname{div} \mathbf{f}_{\varepsilon}\right) d y \\
& +2 \int_{\mathbb{R}^{n}}\left(\left\langle\operatorname{div} \mathbf{f}_{\varepsilon}, \nabla \eta\right\rangle+q_{\varepsilon} \Delta \eta\right) \Phi(x-y) d y \\
:= & -I_{\varepsilon}^{1}(x)-2 I_{\varepsilon}^{2}(x)-I_{\varepsilon}^{3}(x),
\end{aligned}
$$

where

$$
\begin{aligned}
I_{\varepsilon}^{1}(x) & :=\int_{\mathbb{R}^{n}} \eta(y) \Phi(x-y) \operatorname{div}\left(\operatorname{div} \mathbf{f}_{\varepsilon}(y)\right) d y \\
I_{\varepsilon}^{2}(x) & :=\int_{\mathbb{R}^{n}}\left\langle\operatorname{div} \mathbf{f}_{\varepsilon}(y), \nabla \eta(y)\right\rangle \Phi(x-y) d y \\
I_{\varepsilon}^{3}(x) & :=\int_{\mathbb{R}^{n}} q_{\varepsilon}(y) \Delta \eta(y) \Phi(x-y) d y
\end{aligned}
$$


By direct computations, observe that, for $1 \leq i, j \leq n$

$$
\begin{aligned}
(\eta \Phi)_{y_{i}}= & \eta_{y_{i}} \Phi(y)-\frac{1}{\omega_{n}} \frac{\eta y_{i}}{|y|^{n}} \\
(\eta \Phi)_{y_{i} y_{j}}= & \eta_{y_{i} y_{j}} \Phi(y)-\frac{1}{\omega_{n}} \frac{y_{i} \eta_{y_{j}}+y_{j} \eta_{y_{i}}}{|y|^{n}} \\
& -\frac{1}{\omega_{n}}\left(\delta_{i j}-n \frac{y_{i} y_{j}}{|y|^{2}}\right) \frac{\eta}{|y|^{n}}
\end{aligned}
$$

where $\delta_{i j}$ is the Krönecker delta and $\omega_{n}:=n \alpha_{n}$ is the surface area of the unit sphere $\mathbb{S}^{n-1}$. We now establish an uniform local $h^{r}$-estimates $(1 \leq r<\infty)$ for $q_{\varepsilon}$ through the following steps.

Step 1: Limit of $I_{\varepsilon}^{3}$. Let us fix $x \in W \subset \subset V \subset \subset U$. Since $\Delta \eta=0$ on $W$, the integrand in $I_{\varepsilon}^{3}(x)$ is smooth. Since $q_{\varepsilon}$ is determined up to a constant, we can add a constant to $y \mapsto \Delta \eta(y) \Phi|x-y|$, if nessecary, to ensure that it has vanishing integral. For each fixed $x \in W$, let $\mathbf{v}_{x}: V \rightarrow \mathbb{R}^{n}$ be the solution of the Dirichlet problem

$$
\begin{cases}\operatorname{div} \mathbf{v}_{x}(y)=\Delta \eta(y) \Phi(x-y) & \text { for } y \in V \\ \mathbf{v}_{x}=0 & \text { on } \partial V .\end{cases}
$$

Then using (2.19), integrating by parts, and the convergence of $\mathbf{f}_{\varepsilon}$ in (2.16), we obtain

$$
\begin{aligned}
I_{\varepsilon}^{3}(x) & =\int_{\mathbb{R}^{n}} q_{\varepsilon}(y) \Delta \eta(y) \Phi(x-y) d y \\
& =\int_{\mathbb{R}^{n}} q_{\varepsilon}(y) \operatorname{div} \mathbf{v}_{x}(y) d y \\
& =-\int_{\mathbb{R}^{n}}\left\langle\nabla q_{\varepsilon}(y), \mathbf{v}_{x}(y)\right\rangle d x \\
& =-\int_{\mathbb{R}^{n}}\left\langle\operatorname{div} \mathbf{f}_{\varepsilon}(y), \mathbf{v}_{x}(y)\right\rangle d y \\
& \left.=\int_{\mathbb{R}^{n}} \mathbf{f}_{\varepsilon}(y): \nabla_{y} \mathbf{v}_{x}(y)\right\rangle d y \\
& \rightarrow \int_{\mathbb{R}^{n}} \mathbf{f}(y): \nabla_{y} \mathbf{v}_{x}(y) d y \quad \text { as } \varepsilon \rightarrow 0 \\
& =I_{0}^{3}(x) \quad \text { for } x \in W \subset \subset V .
\end{aligned}
$$

Since $\mathbf{f}_{\varepsilon} \rightarrow \mathbf{f}$ strongly in $h^{r}\left(V, \mathbb{M}^{n \times n}\right)$, it follows that $I_{\varepsilon}^{3} \rightarrow I_{0}^{3}$ strongly in $h^{r}(W)$. 
Step 2: Limit of $I_{\varepsilon}^{2}$. Let us fix $x \in W \subset \subset V \subset \subset U$. Integrating by parts, invoking (2.17) and letting $\varepsilon \rightarrow 0$ we have

$$
\begin{aligned}
I_{\varepsilon}^{2}(x) & =\int_{\mathbb{R}^{n}}\left\langle\operatorname{div} \mathbf{f}_{\varepsilon}(y), \Phi(x-y) \nabla \eta(y)\right\rangle d y \\
& =-\int_{\mathbb{R}^{n}} \mathbf{f}_{\varepsilon}: \nabla_{y}(\Phi(x-y) \nabla \eta) d y \\
& =-\int_{\mathbb{R}^{n}} \mathbf{f}_{\varepsilon}:\left(\Phi(x-y) \nabla^{2} \eta-\frac{(y-x) \otimes \nabla \eta}{\omega_{n}|y-x|^{n}}\right) d y \\
& \rightarrow-\int_{\mathbb{R}^{n}} \mathbf{f}:\left(\Phi(x-y) \nabla^{2} \eta-\frac{(y-x) \otimes \nabla \eta}{\omega_{n}|y-x|^{n}}\right) d y \\
& :=I_{0}^{2}(x) \quad x \in W
\end{aligned}
$$

Using the strong convergence of $\mathbf{f}_{\varepsilon}$ in $h^{r}(V)$, again it follows that $I_{\varepsilon}^{2} \rightarrow I_{0}^{2}$ in $h^{r}(W)$.

Step 3: Limit of $I_{\varepsilon}^{1}$. Integrating by parts twice the integral in (2.14) and using (2.18)

$$
\begin{aligned}
I_{\varepsilon}^{1}(x)= & \int_{\mathbb{R}^{n}} \operatorname{div} \operatorname{div} \mathbf{f}_{\varepsilon}(y) \eta(y) \Phi(x-y) d y \\
= & \int_{\mathbb{R}^{n}} \mathbf{f}_{\varepsilon}(y): \nabla_{y}^{2}(\eta(y) \Phi(x-y)) d y \\
= & \int_{\mathbb{R}^{n}} \mathbf{f}_{\varepsilon}(y):\left(\Phi(x-y) \nabla^{2} \eta(y)-\frac{1}{\omega_{n}} \frac{\nabla \eta \otimes(y-x)+(y-x) \otimes \nabla \eta}{|x-y|^{n}}\right) d y \\
& \left.-\frac{1}{\omega_{n}} \int_{\mathbb{R}^{n}} \mathbf{f}_{\varepsilon}(y):\left(I d_{n}-n \frac{(y-x) \otimes(y-x)}{|x-y|^{2}}\right) \frac{\eta}{|x-y|^{n}}\right) d y \\
:= & I_{\varepsilon}^{11}(x)+I_{\varepsilon}^{12}(x), \quad x \in W,
\end{aligned}
$$

where $I d_{n}$ is the $n \times n$ identity matrix. Using the convergence of $\mathbf{f}_{\varepsilon}$, observe that as $\varepsilon \rightarrow 0$,

$$
\begin{aligned}
I_{\varepsilon}^{11}(x) & :=\int_{\mathbb{R}^{n}} \mathbf{f}_{\varepsilon}:\left(\Phi(x-y) \nabla^{2} \eta-\frac{\nabla \eta \otimes(y-x)+(y-x) \otimes \nabla \eta}{\omega_{n}|x-y|^{n}}\right) d y \\
& \rightarrow \int_{\mathbb{R}^{n}} \mathbf{f}:\left(\Phi(x-y) \nabla^{2} \eta-\frac{\nabla \eta \otimes(y-x)+(y-x) \otimes \nabla \eta}{\omega_{n}|x-y|^{n}}\right) d y \\
(2.22) & :=I_{0}^{11}(x), \quad x \in W .
\end{aligned}
$$

In order to estimate $I_{\varepsilon}^{12}$, define the kernels $\Omega_{i j}: \mathbb{R}^{n} \backslash\{0\} \rightarrow \mathbb{R}$ by

$$
\Omega_{i j}(y):=\delta_{i j}-n \frac{y_{i} y_{j}}{|y|^{2}}, \quad y \in \mathbb{R}^{n} \backslash\{0\}, \quad i, j=1, \cdots, n .
$$


Since $n \alpha_{n}=\omega_{n}$, integrating by parts, observe that for any $i, j=1, \cdots, n$,

$$
\begin{aligned}
\int_{\mathbb{S}^{n-1}} \Omega_{i j}(y) d \sigma(y) & =\int_{\mathbb{S}^{n-1}}\left(\delta_{i j}-n y_{i} y_{j}\right) d \sigma(y) \\
& =\omega_{n} \delta_{i j}-n \int_{\mathbb{S}^{n-1}} y_{i} y_{j} d \sigma(y) \\
& =\omega_{n} \delta_{i j}-n \int_{B_{1}} \frac{\partial}{\partial y_{j}} y_{i} d y \\
& =\omega_{n} \delta_{i j}-n \delta_{i j} \alpha_{n} \\
& =0 .
\end{aligned}
$$

Hence each $\Omega_{i j}$ satisfies all the conditions of Calderón-Zygmund Kernel St 70]. Therefore,

$$
\left.I_{\varepsilon}^{12}(x):=-\frac{1}{\omega_{n}} \int_{\mathbb{R}^{n}} \eta \mathbf{f}_{\varepsilon}:\left(I d_{n}-n \frac{(y-x) \otimes(y-x)}{|x-y|^{2}}\right) \frac{d y}{|x-y|^{n}}\right)
$$

is the sum of Calderón-Zygmund type singular integrals with the homogeneous kernel $\Omega_{i j}$. Since $\mathbf{f} \in h^{r}\left(U, \mathbb{M}^{n \times n}\right), 1 \leq r<\infty$, by Theorem 2.1 $I^{12} \in h^{r}(W)$. Furthermore, the following sum of singular integrals

$$
I_{0}^{12}(x):=-\frac{1}{\omega_{n}} \int_{\mathbb{R}^{n}} \eta \mathbf{f}:\left(I d_{n}-n \frac{(y-x) \otimes(y-x)}{|x-y|^{2}}\right) \frac{d y}{|x-y|^{n}}
$$

exists for almost every $x \in W \subset \subset V$ and is in $h^{r}(W)$. From the singular integrals (2.24) and (2.25), by Theorem 2.1, we have

$$
I_{\varepsilon}^{12}(x)-I_{0}^{12}(x)=-\frac{1}{\omega_{n}} \sum_{i, j=1}^{n} \int_{\mathbb{R}^{n}}\left(\eta\left(f_{j}^{i}\right)_{\varepsilon}(y)-\eta f_{j}^{i}(y)\right) \frac{\Omega_{i j}(x-y)}{|x-y|^{n}} d y
$$

Hence there exists $C:=C(V, W, r)>0$ such that

$$
\left\|I_{\varepsilon}^{12}-I_{0}^{12}\right\|_{h^{r}(W)} \leq C \sum_{j=1}^{n}\left\|\left(f_{j}^{i}\right)_{\varepsilon}-f_{j}^{i}\right\|_{h^{r}(V)} \rightarrow 0 \quad \text { as } \varepsilon \rightarrow 0 .
$$

Step 4: Explicit representation of $q$. To complete the proof, let us define the potential $q: W \rightarrow \mathbb{R}$ by

$$
q(x):=-\left(I_{0}^{11}(x)+I_{0}^{12}(x)+2 I_{0}^{2}(x)+I_{0}^{3}(x)\right) .
$$


Then from (2.20), (2.21), (2.22), and (2.26), we conclude that $q_{\varepsilon} \rightarrow q$ strongly in $h_{\mathrm{loc}}^{r}$ for any $1 \leq r<\infty$, and hence and $q$ is represented as

$$
\begin{aligned}
q(x)= & \int_{U} \mathbf{f}:\left(\Phi(x-y) \nabla^{2} \eta-\nabla_{y} \mathbf{v}_{x}\right) d y \\
& +\frac{1}{\omega_{n}} \int_{U} \mathbf{f}:(\nabla \eta \otimes(y-x)-(y-x) \otimes \nabla \eta) \frac{d y}{|x-y|^{n}} \\
& +\frac{1}{\omega_{n}} \int_{U} \eta \mathbf{f}:\left(I d_{n}-n \frac{(y-x) \otimes(y-x)}{|x-y|^{2}}\right) \frac{d y}{|x-y|^{n}}
\end{aligned}
$$

for any $x \in W$. Since $q$ is the strong limit of the family $q_{\varepsilon}$ in $W$, it is independent of the choice of the cut-off function $\eta$. This completes the proof of Theorem 1.1.

\section{First Variation of Energy and the existence of HYDROSTATIC PRESSURE}

Let $\Omega \subset \mathbb{R}^{n}, n \geq 2$ be a smooth, simply connected and bounded domain and let $L: \mathbb{M}^{n \times n} \rightarrow \mathbb{R}$ be smooth function. We are now in a position to establish the existence of integrable hydrostatic pressure associated with the local minimizers of the energy

$$
E[\mathbf{w}]:=\int_{\Omega} L(\nabla \mathbf{w}(x)) d x
$$

for incompressible $W^{1,2}$-deformations $\mathbf{w}: \Omega \subset \mathbb{R}^{n} \rightarrow \mathbb{R}^{n}$. By direct computation, observe that Mooney-Rivlin bulk-energy given by

$$
L(X)=\frac{\mu_{1}}{2}\left(|\nabla \mathbf{u}|^{2}-3\right)+\frac{\mu_{2}}{2}\left(|\operatorname{cof} \nabla \mathbf{u}|^{2}-3\right),
$$

satisfies the following.

$$
D L=\mu_{1} P+\mu_{2}\left(\begin{array}{ccc}
\operatorname{cof}(S Q)_{1}^{1}:(S P)_{1}^{1} & -\operatorname{cof}(S Q)_{2}^{1}:(S Q)_{2}^{1} & \operatorname{cof}(S Q)_{3}^{1}:(S P)_{3}^{1} \\
-\operatorname{cof}(S Q)_{1}^{2}:(S P)_{1}^{2} & \operatorname{cof}(S Q)_{2}^{2}:(S P)_{2}^{2} & -\operatorname{cof}(S Q)_{3}^{2}:(S P)_{3}^{2} \\
\operatorname{cof}(S Q)_{1}^{3}:(S P)_{1}^{3} & -\operatorname{cof}(S Q)_{2}^{3}:(S P)_{2}^{3} & \operatorname{cof}(S Q)_{3}^{3}:(S P)_{3}^{3}
\end{array}\right),
$$

where $Q:=\operatorname{cof} P$, and $(S X)_{j}^{i}$ is the $2 \times 2$ submatrix obtained by deleting the $i$-th row and the $j$-th column of the matrix $X \in M^{3 \times 3}$. Furthermore, 
the Cauchy-Green strain tensor is given by

$$
(D L(P))^{t} P=\mu_{1} P^{t} P+\mu_{2}\left(\begin{array}{ccc}
\left|Q_{2}\right|^{2}+\left|Q_{3}\right|^{2} & -\left\langle Q_{1}, Q_{2}\right\rangle & -\left\langle Q_{1}, Q_{3}\right\rangle \\
-\left\langle Q_{1}, Q_{2}\right\rangle & \left|Q_{1}\right|^{2}+\left|Q_{3}\right|^{2} & -\left\langle Q_{2}, Q_{3}\right\rangle \\
-\left\langle Q_{1}, Q_{2}\right\rangle & -\left\langle Q_{2}, Q_{3}\right\rangle & \left|Q_{1}\right|^{2}+\left|Q_{2}\right|^{2}
\end{array}\right)
$$

for all $P \in \mathbb{M}^{3 \times 3}$, where $Q_{i}:=(\operatorname{cof} P)_{i}:=\left((\operatorname{cof} P)_{1}^{i},(\operatorname{cof} P)_{2}^{i},(\operatorname{cof} P)_{3}^{i}\right)$ be the $i$-th row of $\operatorname{cof} P, i=1,2,3$. Motivated by the above calculations, assume that $L$ satisfies the following growth condition.

$$
\max \left(|L(P)|,\left|(D L(P))^{t} P\right|\right) \leq C\left(1+|P|^{2}+|\operatorname{cof} P|^{2}\right)
$$

for some $C>0$, for any $P \in \mathbb{M}^{n \times n}$.

Now we prove the existence of an integrable hydrostatic pressure $q$ on the deformed domain $\mathbf{u}(\Omega)$ and establish an explicit representation of the pressure $q$ in terms of Calderón-Zygmund type singular integrals of the Cauchy-Green strain $\left.\tilde{\sigma}:=(D L(\nabla \mathbf{u}))^{t} \nabla \mathbf{u}\right) \circ \mathbf{u}^{-1}$ in $\mathbf{u}(\Omega)$. Our proof consists of deriving the first variation of the energy $E[\cdot]$, obtaining the equation $\nabla q=\operatorname{div} \tilde{\sigma}$, and then finally use Theorem 2.2 .

Theorem 3.1. Let $L: \mathbb{M}^{n \times n} \rightarrow \mathbb{R}$ be smooth and satisfies the growth condition (3.3). Assume that $\boldsymbol{u} \in \mathcal{A}$ be a continuous and injective local minimizer of $E[\cdot]$, such that $|\nabla \boldsymbol{u}|^{2},|\operatorname{cof} \nabla \boldsymbol{u}|^{2} \in h_{\mathrm{loc}}^{r}(\Omega)$ for some $1 \leq r<$ $\infty$. Then there exists a scalar function $q \in h_{\mathrm{loc}}^{r}(\boldsymbol{u}(\Omega))$, such that

$\|q\|_{h^{r}(V)} \leq C\left(\left\||\nabla \boldsymbol{u}|^{2}\right\|_{h^{r}\left(\boldsymbol{u}^{-1}(V)\right)}+\left\||\operatorname{cof} \nabla \boldsymbol{u}|^{2}\right\|_{h^{r}\left(\boldsymbol{u}^{-1}(V)\right)}\right), \quad V \subset \subset \boldsymbol{u}(\Omega)$,

for some $C>0$ (depending on $r, V, n$ and $\boldsymbol{u}(\Omega)$ ) and the pair $(\boldsymbol{u}, q)$ satisfies the integral identity

$$
\int_{\Omega} D L(\nabla \boldsymbol{u}(x)): \nabla(\boldsymbol{v} \circ \boldsymbol{u}) d x=\int_{u(\Omega)} q(y) \operatorname{div} \boldsymbol{v}(y) d y
$$

for all $\boldsymbol{v} \in C_{0}^{\infty}\left(\boldsymbol{u}(\Omega), \mathbb{R}^{n}\right)$, where $A: B:=\operatorname{tr}\left(\mathrm{A}^{\mathrm{t}} \mathrm{B}\right)=\sum_{\mathrm{i}, \mathrm{j}=1}^{\mathrm{n}} \mathrm{a}_{\mathrm{j}}^{\mathrm{i}} \mathrm{b}_{\mathrm{j}}^{\mathrm{j}}$ is the scalar product on $\mathbb{M}^{n \times n}$. 
Remark 3.2. Let $W \subset \subset V \subset \subset \mathbf{u}(\Omega)$, and $\eta \in C_{0}^{\infty}(V)$ be a cut-off function such that $\eta \equiv 1$ on $W$. Then $q$ is locally represented as

$$
\begin{aligned}
q(x)= & \int_{V} \tilde{\sigma}:\left(\Phi(x-y) \nabla^{2} \eta-\nabla_{y} \mathbf{v}_{x}\right) d y \\
& +\frac{1}{\omega_{n}} \int_{V} \tilde{\sigma}:(\nabla \eta \otimes(y-x)-(y-x) \otimes \nabla \eta) \frac{d y}{|x-y|^{n}} \\
& +\frac{1}{\omega_{n}} \int_{V} \eta \tilde{\sigma}:\left(I d_{n}-n \frac{(y-x) \otimes(y-x)}{|x-y|^{2}}\right) \frac{d y}{|x-y|^{n}}
\end{aligned}
$$

for any $x \in W$, where $\Phi$ is Newtonian potential in $\mathbb{R}^{n}$ defined in (2.12) and $\mathbf{v}_{x}$ as defined in (2.19).

Remark 3.3. In the study of regularity of finite energy deformations, Šverák Sv 88] proved that for any $W^{1, n}$-deformation $\mathbf{w}$ with det $\nabla \mathbf{w}(x)>$ 0 , a.e., there exists a continuous function $\omega$ on $\mathbb{R}$ with $\omega(0)=0$ such that

$$
|\mathbf{w}(x)-\mathbf{w}(y)| \leq \omega(|x-y|), \quad \text { for any } x, y \in \Omega \subset \subset \mathbb{R}^{n} .
$$

It is also well-known any $W^{1, n}$-deformation $\mathbf{w}$ for which the distortion function $K(\cdot, \mathbf{w}):=|\nabla \mathbf{w}(\cdot)|^{n} / \operatorname{det} \nabla \mathbf{w}(\cdot) \in L^{r}$ for some $r>n-1$, is a homeomorphism. Thus in particular, area-preserving $W^{1, r}(r>2)$ deformations in the plane are continuous and open maps. However, in general for $n \geq 3$, any deformation $\mathbf{w} \in K^{1,2}$ may be totally discontinuous, see [Sv 88, p. 119].

In order to prove Theorem 3.1, we establish the following first variation of the energy integral $E[\cdot]$.

Lemma 3.4. First Variation. Let $\boldsymbol{u} \in \mathcal{A}$ be a local minimizer of $E[\cdot]$. We further assume that $\boldsymbol{u}$ is a continuous and an injective map. Then $\boldsymbol{u}$ satisfies the following integral identity

$$
\int_{\Omega} D L(\nabla \boldsymbol{u}(x)): \nabla(\boldsymbol{v} \circ \boldsymbol{u})(x) d x=0,
$$

for all smooth, compactly supported and divergence free vector fields $\boldsymbol{v}$ on $\boldsymbol{u}(\Omega)$.

Proof: By the invariance of domain $\mathbf{u}(\Omega)$ is open and $\mathbf{u}: \Omega \rightarrow \mathbf{u}(\Omega)$ is a homeomorphism. Let $\mathbf{v} \in C_{0}^{\infty}\left(\mathbf{u}(\Omega), \mathbb{R}^{n}\right)$ be a vector field with $\operatorname{div} \mathbf{v}=0$. For each $y \in \mathbf{u}(\Omega)$, consider the unique smooth flow $\phi(y, \cdot): \mathbb{R} \rightarrow \mathbf{u}(\Omega)$ given by

$$
\frac{d \phi}{d t}(y, t)=\mathbf{v}(\phi(y, t)) \quad \text { in } \quad \mathbb{R}, \quad \phi(y, 0)=y
$$


Using the relations $\frac{\partial}{\partial P_{j}^{i}} \operatorname{det} P=(\operatorname{cof} P)_{j}^{i}$ and $P(\operatorname{cof} P)^{t}=I d_{n} \operatorname{det} P$, by a direct calculations we observe that

$$
\frac{d}{d t}\left(\operatorname{det} \nabla_{y} \phi(y, t)\right)=\operatorname{det} \nabla_{y} \phi(y, t) \operatorname{div} \mathbf{v}=0 .
$$

Since det $\nabla_{y} \phi(y, 0)=1$, from (3.8) it follows that $\operatorname{det} \nabla_{y} \phi(y, t)=1$ for all $t \in \mathbb{R}$ and $y \in \mathbf{u}(\Omega)$. Consider the map $\mathbf{w}: \Omega \times \mathbb{R} \rightarrow \mathbf{u}(\Omega)$ defined by

$$
\mathbf{w}(x, t):=\phi(\cdot, t) \circ \mathbf{u}(x)=\phi(\mathbf{u}(x), t) \quad \text { for any } t \in \mathbb{R}, x \in \Omega .
$$

Let $V:=\operatorname{supp} \mathbf{v} \subset \mathbf{u}(\Omega)$, then $\mathbf{v}(\mathbf{u}(x))=0$ for $\mathbf{u}(x) \notin V$. This in conjunction with the uniqueness of $\phi$ implies that $\phi(\mathbf{u}(x), t)=\mathbf{u}(x)$ for all points $x$ such that $\mathbf{u}(x) \notin V$. Since $\Omega$ is bounded, $\mathbf{u}$ is continuous and $V$ is compact, $\Omega^{\prime}=\mathbf{u}^{-1}(V)$ is a compact subset of $\Omega$. Hence $\operatorname{supp}(\mathbf{w}(x, t)-$ $\mathbf{u}(x)) \subset \Omega^{\prime}$. Furthermore, $\operatorname{det} \nabla_{x} \mathbf{w}(x, t)=\operatorname{det} \nabla_{y} \phi(y, t) \operatorname{det} \nabla \mathbf{u}(x)=1$. Therefore, $\mathbf{w}(\cdot, t) \in \mathcal{A}$ and $\operatorname{supp}(\mathbf{u}-\mathbf{w}(\cdot, t)) \subset \Omega$ for all $t \in \mathbb{R}$. Since $\mathbf{u}$ is a local minimizer of $E[\cdot]$,

$$
E[\mathbf{u}] \leq E[\mathbf{w}(\cdot, t)] \quad \text { for all } \quad t \in \mathbb{R}
$$

Thus in particular,

$$
\begin{aligned}
0 & =\left.\frac{d}{d t} \int_{\Omega} L(\nabla \mathbf{w}(x, t)) d x\right|_{t=0} \\
& =\left.\sum_{i, j=1}^{2} \int_{\Omega} L_{j}^{i}(\nabla \mathbf{w}(x, t)) \frac{d}{d t}\left(\frac{\partial w^{i}}{\partial x_{j}}(x, t)\right) d x\right|_{t=0} \\
& =\left.\sum_{i, j=1}^{2} \int_{\Omega} L_{j}^{i}(\nabla \mathbf{w}(x, t)) \frac{\partial}{\partial x_{j}}\left(\frac{d \phi^{i}}{d t}(\mathbf{u}(x), t)\right) d x\right|_{t=0} \\
& =\sum_{i, j=1}^{2} \int_{\Omega} L_{j}^{i}(\nabla \mathbf{w}(x, t)) \frac{\partial}{\partial x_{j}}\left(\left.v^{i}(\phi(u(x), t)) d x\right|_{t=0}\right. \\
& =\sum_{i, j=1}^{2} \int_{\Omega} L_{j}^{i}(\nabla \mathbf{u}(x)) \frac{\partial}{\partial x_{j}}\left(v^{i}(\mathbf{u}(x))\right) d x \\
& =\int_{\Omega} D L(\nabla \mathbf{u}(x)): \nabla(\mathbf{v} \circ \mathbf{u})(x) d x
\end{aligned}
$$

for all smooth, compactly supported and divergence free vector fields on $\mathbf{u}(\Omega)$, where $L_{j}^{i}(P):=\frac{\partial L}{\partial p_{j}^{i}}(P)$. This proves the Theorem. 
Proof of Theorem 3.1: Let $1 \leq r<\infty$ and $U^{\prime} \subset \subset U$. Let $\mathbf{u} \in \mathcal{A}$ be a local minimizer of $E[\cdot]$ such that $|\nabla \mathbf{u}|^{2} \in h^{r}$ and $|\operatorname{cof} \nabla \mathbf{u}|^{2} \in h^{r}\left(U^{\prime}\right)$ for some $1 \leq r<\infty$. Assume further that $\mathbf{u}: \Omega \rightarrow \mathbf{u}(\Omega)$ is continuous and bijective map.

Now define $\mathbf{g}=\left(g^{1}, \cdots, g^{n}\right): C_{0}^{1}\left(\mathbf{u}(\Omega), \mathbb{R}^{n}\right) \rightarrow \mathbb{R}$ by

$$
\langle\mathbf{g}, \mathbf{v}\rangle:=\int_{\Omega} D L(\nabla \mathbf{u}(x)): \nabla(\mathbf{v} \circ \mathbf{u})(x) d x,
$$

for all $\mathbf{v}=\left(v^{1}, \cdots, v^{n}\right) \in C_{0}^{1}\left(\mathbf{u}(\Omega), \mathbb{R}^{n}\right)$. In view of the volume constraint and growth condition (3.3), it follows that

$$
|\langle\mathbf{g}, \mathbf{v}\rangle| \leq C\left(1+\|\nabla u\|_{L^{2}(\Omega)}+\|\operatorname{cof} \nabla u\|_{L^{2}(\Omega)}\right)\|\nabla \mathbf{v}\|_{L^{\infty}(\mathbf{u}(\Omega))},
$$

for any $\mathbf{v} \in C_{0}^{1}\left(\mathbf{u}(\Omega), \mathbb{R}^{n}\right)$. Hence $\mathbf{g}$ is a continuous linear functional on $C_{0}^{1}\left(\mathbf{u}(\Omega), \mathbb{R}^{n}\right)$. Using the the first variation (3.6), we conclude that

$$
\langle\mathbf{g}, \mathbf{v}\rangle=0 \quad \forall \mathbf{v} \in C_{0}^{1}\left(\mathbf{u}(\Omega), \mathbb{R}^{n}\right), \operatorname{div} \mathbf{v}=0 .
$$

Hence there exists $q \in \mathcal{D}^{\prime}(\mathbf{u}(\Omega))$ ( see [Te 01, Proposition 1.1, p10]), such that

$$
\mathbf{g}=-\nabla q \quad \text { in } \mathcal{D}^{\prime}\left(\mathbf{u}(\Omega), \mathbb{R}^{n}\right)
$$

modulo translation of a constant. In order to obtain $h^{r}$ estimates of $q$, for $1 \leq i, j \leq n$, let us define $\sigma_{j}^{i}: \Omega \rightarrow \mathbb{R}$ by

$$
\sigma_{j}^{i}(x):=\sum_{k=1}^{n} L_{k}^{i}(\nabla \mathbf{u}(x)) \frac{\partial u^{j}}{\partial x_{k}}(x) \quad \text { for } x \in \Omega,
$$

so that, the Cauchy-Green strain tensor on $\Omega$ is given by

$$
\sigma:=\left(\sigma_{j}^{i}\right)=(D L(\nabla \mathbf{u}))^{t} \nabla \mathbf{u}
$$

Define the $i j$-th component of the Cauchy-Green Strain tensor $\tilde{\sigma}_{j}^{i}$ on the deformed domain $\mathbf{u}(\Omega)$ by

$$
\tilde{\sigma}_{j}^{i}:=\sigma_{j}^{i} \circ \mathbf{u}^{-1} \quad \text { on } \mathbf{u}(\Omega), i, j=1, \cdots, n .
$$

The growth condition $\left|\sigma_{j}^{i}\right| \leq C\left(|\nabla \mathbf{u}|^{2}+|\operatorname{cof} \nabla \mathbf{u}|^{2}\right)$ and $|\nabla \mathbf{u}|^{2},|\operatorname{cof} \nabla \mathbf{u}|^{2} \in$ $L \log L$ yields $\tilde{\sigma}_{j}^{i} \in h^{1}(V)$. If $\mathbf{u} \in K_{\mathrm{loc}}^{1,2 r}\left(\Omega, \mathbb{R}^{n}\right), 1<r<\infty$, from the definition of $\sigma_{j}^{i}, \tilde{\sigma}_{j}^{i}$, and the condition (3.3) on $L$, it follows that

$$
\begin{aligned}
\int_{V} \mid\left(\left.\tilde{\sigma}_{j}^{i}\right|^{r}\right. & =\int_{\mathbf{u}^{-1}(V)}\left|\sigma_{j}^{i}\right|^{r} \\
& \leq C\left(\|\nabla \mathbf{u}\|_{L^{2 r}\left(\mathbf{u}^{-1}(V)\right)}^{2 r}+\|\operatorname{cof} \nabla \mathbf{u}\|_{L^{2 r}\left(\mathbf{u}^{-1}(V)\right)}^{2 r}\right),
\end{aligned}
$$


for any $V \subset \subset \mathbf{u}(\Omega)$. Therefore, if $|\nabla \mathbf{u}|^{2} \in h^{r}$ and $|\operatorname{cof} \nabla \mathbf{u}|^{2} \in h_{\text {loc }}^{r}$ for some $1 \leq r<\infty$, from (3.16), we have

$$
\sigma:=\left(\sigma_{j}^{i}\right) \in h_{\mathrm{loc}}^{r}\left(\Omega, \mathbb{M}^{n \times n}\right) \quad \text { and } \quad \tilde{\sigma}:=\left(\tilde{\sigma}_{j}^{i}\right) \in h_{\mathrm{loc}}^{r}\left(\mathbf{u}(\Omega), \mathbb{M}^{n \times n}\right) .
$$

Observe that, from the definition of $\mathbf{g}$ in (3.9), $\sigma_{j}^{i}$ in (3.13), $\tilde{\sigma}_{j}^{i}$ in (3.15), and change of variables,

$$
\begin{aligned}
\langle\mathbf{g}, \mathbf{v}\rangle & =\sum_{i, k=1}^{n} \int_{\Omega} L_{k}^{i}(\nabla \mathbf{u}(x)) \frac{\partial}{\partial x_{k}}\left(v^{i} \circ \mathbf{u}\right)(x) d x \\
& =\sum_{i, j, k=1}^{n} \int_{\Omega} L_{k}^{i}(\nabla \mathbf{u}(x)) \frac{\partial v^{i}}{\partial y_{j}}(\mathbf{u}(x)) \frac{\partial u^{j}}{\partial x_{k}}(x) d x \\
& =\sum_{i, j=1}^{n} \int_{\Omega} \sigma_{j}^{i}(x) \frac{\partial v^{i}}{\partial y_{j}}(\mathbf{u}(x)) d x \\
& =\int_{\Omega} \sigma(x): \nabla_{\mathbf{u}} \mathbf{v}(\mathbf{u}(x)) d x \\
& =\int_{\mathbf{u}(\Omega)} \tilde{\sigma}(y): \nabla \mathbf{v}(y) d y \\
& =-\langle\operatorname{div} \tilde{\sigma}, \mathbf{v}\rangle
\end{aligned}
$$

for any $v \in C_{0}^{1}\left(\mathbf{u}(\Omega), \mathbb{R}^{n}\right)$. Hence

$$
\mathbf{g}=-\operatorname{div} \tilde{\sigma} \quad \text { in } \mathcal{D}^{\prime}\left(\mathbf{u}(\Omega), \mathbb{M}^{n \times n}\right)
$$

where the divergence is taken in each rows. Therefore, combining (3.12) and (3.18), we get

$$
\nabla q=\operatorname{div} \tilde{\sigma} \quad \text { in } \mathcal{D}^{\prime}\left(\mathbf{u}(\Omega), \mathbb{M}^{n \times n}\right) .
$$

By taking $\mathbf{f}=\tilde{\sigma}$, and $U=V \subset \subset \mathbf{u}(\Omega)$ in (3.19), from Theorem 2.2, we conclude that $q \in h_{\mathrm{loc}}^{r}(\mathbf{u}(\Omega))$, it satisfies the local representation (3.5), and

$$
\begin{aligned}
\|q\|_{h^{r}(V)} & \leq C\|\tilde{\sigma}\|_{h^{r}(V)} \\
& \leq C\left(\left\||\nabla \mathbf{u}|^{2}\right\|_{h^{r}\left(\mathbf{u}^{-1}(V)\right)}+\left\||\operatorname{cof} \nabla \mathbf{u}|^{2}\right\|_{h^{r}\left(\mathbf{u}^{-1}(V)\right)}\right),
\end{aligned}
$$

for any $V \subset \subset \mathbf{u}(\Omega)$, for some $C>0$, depending on $r, V, n$ and $\mathbf{u}(\Omega)$. Since $q \in L_{\text {loc }}^{1}$, from (3.12), it follows that

$$
\langle\mathbf{g}, \mathbf{v}\rangle=-\langle\nabla q, \mathbf{v}\rangle=\langle q, \operatorname{div} \mathbf{v}\rangle=\int_{\mathbf{u}(\Omega)} q(y) \operatorname{div} \mathbf{v}(y) d y
$$


for any $\mathbf{v} \in C_{0}^{1}\left(\mathbf{u}(\Omega), \mathbb{R}^{n}\right)$. Hence

$$
\int_{\Omega} D L(\nabla \mathbf{u}(x)): \nabla(\mathbf{v} \circ \mathbf{u})(x) d x=\int_{\mathbf{u}(\Omega)} q(y) \operatorname{div} \mathbf{v}(y) d y,
$$

for any $\mathbf{v} \in C_{0}^{1}\left(\mathbf{u}(\Omega), \mathbb{R}^{n}\right)$. This proves the Theorem.

\section{Derivation of Euler-Lagrange Equations}

Theorem 4.1. Let $\Omega \subset \mathbb{R}^{n}, n \geq 2$, be a smooth, simply connected and bounded domain. Let $\boldsymbol{u} \in \mathcal{A} \cap K_{\mathrm{loc}}^{1, s}\left(\Omega, \mathbb{R}^{n}\right)$ for some $s \geq 3$ be a continuous and injective local minimizer of $E[\cdot]$. Then the hydrostatic pressure $p:=q \circ \boldsymbol{u} \in L_{\mathrm{loc}}^{s / 2}(\Omega)$, and the pair $(\boldsymbol{u}, p)$ satisfies

$$
\int_{\Omega} D L(\nabla \boldsymbol{u}(x)): \nabla \phi(x) d x=\int_{\Omega} p(x) \operatorname{cof}(\nabla \boldsymbol{u}(x)): \nabla \phi(x) d x,
$$

for all $\phi \in C_{0}^{1}\left(\Omega, \mathbb{R}^{n}\right)$, where $q \in L_{\text {loc }}^{s / 2}(\boldsymbol{u}(\Omega))$ as in Theorem 3.1. In other words, the pair $(\boldsymbol{u}, p)$ satisfies the system of Euler-Lagrange equations

$$
\operatorname{div}[D L(\nabla \boldsymbol{u}(x))-p(x) \operatorname{cof}(\nabla \boldsymbol{u}(x))]=0 \quad \text { in } \Omega,
$$

in the sense of distribution, where the divergence is taken in each rows.

Proof. Let $\Omega \subset \mathbb{R}^{n}$ be a smooth, simply connected domain. Recall that $K^{1, s}:=\left\{\mathbf{w} \in W^{1, s}: \operatorname{cof} \nabla \mathbf{w} \in L^{s}\right\}$ and $\mathcal{A}:=\left\{\mathbf{w} \in K^{1,2}\left(\Omega, \mathbb{R}^{n}\right)\right.$ : $\operatorname{det} \nabla \mathbf{w}=1$ a.e. $\}$. Let $\mathbf{u} \in \mathcal{A} \cap K_{\text {loc }}^{1, s}\left(\Omega, \mathbb{R}^{n}\right), s \geq 3$ be a continuous injective local minimizer of the functional $E[\cdot]$. By Theorem 3.1 , there exists $q \in L_{\text {loc }}^{s / 2}$ such that the pair $(\mathbf{u}, q)$ satisfies the identity (3.21). Let $\mathbf{u}^{-1}: \mathbf{u}(\Omega) \rightarrow \Omega$ be the inverse of $\mathbf{u}$. Then using the volume-constraint we obtain

$$
\nabla_{y} \mathbf{u}^{-1}(y)=\left(\nabla_{x} \mathbf{u}(x)\right)^{-1}=(\operatorname{cof} \nabla \mathbf{u}(x))^{t}, \quad y=\mathbf{u}(x),
$$

and hence by the change of variables

$$
\int_{\mathbf{u}(\Omega)}\left|\nabla \mathbf{u}^{-1}(y)\right|^{2} d y=\int_{\Omega}|\operatorname{cof} \nabla \mathbf{u}(x)|^{2} d x<\infty .
$$

Using the relation $\operatorname{cof}(X Y)=\operatorname{cof} X \operatorname{cof} Y$, for $X, Y \in \mathbb{M}^{n \times n}$, observe that

$$
I d_{n}=\operatorname{cof}\left(\nabla_{y} \mathbf{u}^{-1} \nabla \mathbf{u}\right)=\operatorname{cof}\left(\nabla_{y} \mathbf{u}^{-1}\right) \operatorname{cof}(\nabla \mathbf{u})=\operatorname{cof}\left(\nabla_{y} \mathbf{u}^{-1}\right)(\nabla \mathbf{u})^{-t},
$$

and hence

$$
\operatorname{cof}\left(\nabla \mathbf{u}^{-1}\right)=(\nabla \mathbf{u})^{t}
$$

Since $\mathbf{u} \in K_{\text {loc }}^{1, s}\left(\Omega, \mathbb{R}^{n}\right)$, it follows that $\mathbf{u}^{-1} \in K_{\text {loc }}^{1, s}(\mathbf{u}(\Omega), \Omega)$ for $s \geq 3$. Let $V \subset \subset \mathbf{u}(\Omega)$ and $\phi \in C_{0}^{1}\left(\mathbf{u}^{-1}(V), \mathbb{R}^{n}\right)$. Then the composition $\phi \circ \mathbf{u}^{-1} \in$ 
$W_{0}^{1, s}\left(V, \mathbb{R}^{n}\right)$. Hence there exists $\mathbf{v}_{\varepsilon} \in C_{0}^{1}\left(V, \mathbb{R}^{n}\right)$ such that $\mathbf{v}_{\varepsilon} \rightarrow \psi:=$ $\phi \circ \mathbf{u}^{-1}$ strongly in $W^{1, s}\left(V, \mathbb{R}^{n}\right)$ as $\varepsilon \rightarrow 0$. Let $U:=\mathbf{u}^{-1}(V)$. Then Hölder inequality yields

$$
\begin{aligned}
\int_{U} D L(\nabla \mathbf{u}):\left(\nabla\left(\mathbf{v}_{\varepsilon} \circ \mathbf{u}\right)\right. & -\nabla(\psi \circ \mathbf{u})) d x \\
& =\int_{U}(\nabla \mathbf{u})^{t} D L(\nabla \mathbf{u}):\left(\nabla_{z} \mathbf{v}_{\varepsilon}(\mathbf{u})-\nabla_{z} \psi(\mathbf{u})\right) d x \\
& \leq C\|\nabla \mathbf{u}\|_{L^{2 s^{\prime}}(U)}\left\|\nabla\left(\mathbf{v}_{\varepsilon}-\psi\right)\right\|_{L^{s}(V)}
\end{aligned}
$$

where $s^{\prime}:=s /(s-1)$. Notice that $s \geq 3$ yields $2 s^{\prime} \leq s$ and hence $\nabla \mathbf{u} \in L_{\mathrm{loc}}^{s}(\Omega) \subseteq L_{\mathrm{loc}}^{2 s^{\prime}}(\Omega)$. Therefore, from (3.9) we obtain

$$
\begin{aligned}
\left\langle\mathbf{g}, \mathbf{v}_{\varepsilon}\right\rangle & =\int_{\mathbf{u}^{-1}(V)} D L(\nabla \mathbf{u}(x)): \nabla\left(\mathbf{v}_{\varepsilon} \circ \mathbf{u}\right)(x) d x \\
& \rightarrow \int_{\mathbf{u}^{-1}(V)} D L(\nabla \mathbf{u}(x)): \nabla\left(\phi \circ \mathbf{u}^{-1} \circ \mathbf{u}\right)(x) d x \quad \text { as } \varepsilon \rightarrow 0 \\
& =\int_{\mathbf{u}^{-1}(V)} D L(\nabla \mathbf{u}(x)): \nabla \phi(x) d x .
\end{aligned}
$$

Since $\nabla \mathbf{u}$, cof $\nabla \mathbf{u} \in L_{\mathrm{loc}}^{s}, q \in L_{\mathrm{loc}}^{s / 2}$ and $L_{\mathrm{loc}}^{s / 2} \subseteq L_{\mathrm{loc}}^{s /(s-1)}$ for $s \geq 3$, applying change of variables in (3.21), and letting $\varepsilon \rightarrow 0$ we obtain

$$
\begin{aligned}
\left\langle\mathbf{g}, \mathbf{v}_{\varepsilon}\right\rangle & =\int_{V} q(y) \operatorname{trace}\left(\nabla \mathbf{v}_{\varepsilon}(y)\right) d y \\
& =\int_{\mathbf{u}^{-1}(V)} q(\mathbf{u}(x)) \operatorname{trace}\left(\nabla_{\mathbf{u}} \mathbf{v}_{\varepsilon}(\mathbf{u}(x))\right) d y \\
& =\int_{\mathbf{u}^{-1}(V)} q(\mathbf{u}(x)) \operatorname{trace}\left(\nabla\left(\mathbf{v}_{\varepsilon} \circ \mathbf{u}\right)(x)(\operatorname{cof} \nabla \mathbf{u}(x))^{t}\right) d x \\
& =\int_{\mathbf{u}^{-1}(V)} q(\mathbf{u}(x)) \operatorname{cof}(\nabla \mathbf{u}(x)): \nabla\left(\mathbf{v}_{\varepsilon} \circ \mathbf{u}\right)(x) d x \\
& \rightarrow \int_{\mathbf{u}^{-1}(V)} q(\mathbf{u}(x)) \operatorname{cof}(\nabla \mathbf{u}(x)): \nabla\left(\phi \circ \mathbf{u}^{-1} \circ \mathbf{u}\right)(x) d x \\
= & \int_{\mathbf{u}^{-1}(V)} q(\mathbf{u}(x)) \operatorname{cof}(\nabla \mathbf{u}(x)): \nabla \phi(x) d x
\end{aligned}
$$

Hence from (4.2) and (4.3) we obtain

$$
\int_{\mathbf{u}^{-1}(V)} D L(\nabla \mathbf{u}(x)): \nabla \phi(x) d x=\int_{\mathbf{u}^{-1}(V)} q(\mathbf{u}(x)) \operatorname{cof}(\nabla \mathbf{u}(x)): \nabla \phi(x) d x,
$$


for any $\phi \in C_{0}^{1}\left(\mathbf{u}^{-1}(V), \mathbb{R}^{n}\right)$. Finally choose a sequence of smooth, simply connected sets $V_{k} \subset \subset V_{k+1} \subset \subset \mathbf{u}(\Omega)$ sub-domains such that $\mathbf{u}(\Omega)=$ $\cup_{k=1}^{\infty} V_{k}$. Utilizing the foregoing arguments, there exists $q_{k} \in L^{s / 2}\left(V_{k}\right)$, $k \geq 1$ such that

$$
\int_{\mathbf{u}^{-1}\left(V_{k}\right)} D L(\nabla \mathbf{u}): \nabla \phi=\int_{\mathbf{u}^{-1}\left(V_{k}\right)} q_{k}(\mathbf{u}) \operatorname{cof}(\nabla \mathbf{u}): \nabla \phi,
$$

for $\phi \in C_{0}^{1}\left(\mathbf{u}^{-1}\left(V_{k}\right), \mathbb{R}^{n}\right)$. Since $\mathbf{u}$ is locally volume-preserving homeomorphism, $\Omega=\cup_{k=1}^{\infty} \mathbf{u}^{-1}\left(V_{k}\right)$ is an open covering of $\Omega$ and $\mathbf{u}^{-1}\left(V_{k}\right) \subset \subset$ $\mathbf{u}^{-1}\left(V_{k+1}\right)$. Using the identity div cof $\nabla \mathbf{u}(x)=0$ and invertibility of $\nabla \mathbf{u}(x)$, from (4.4) it follows that $q_{k}$ is unique up to a translation of a constant. Thus adding constant terms as necessary to each $q_{k}$, we deduce from (4.4) that for each fixed $k \geq 1$

$$
q_{i}(z)=q_{k}(z) \quad \text { for } z \in V_{i}, \quad 1 \leq i \leq k .
$$

We finally define $q: \mathbf{u}(\Omega) \rightarrow \mathbb{R}$ as $q(z):=q_{k}(z)$, for $z \in V_{k}$, so that $q \in L_{\text {loc }}^{s / 2}(\mathbf{u}(\Omega))$. This proves that for any $\phi \in C_{0}^{1}\left(\Omega, \mathbb{R}^{n}\right)$, the pair $(\mathbf{u}, q)$ satisfies

$$
\int_{\Omega} D L(\nabla \mathbf{u}(x)): \nabla \phi(x) d x=\int_{\Omega} q(\mathbf{u}(x)) \operatorname{cof}(\nabla \mathbf{u}(x)): \nabla \phi(x) d x .
$$

Now let us define the pressure $p$ on $\Omega$ by

$$
p(x):=q(\mathbf{u}(x)) \text { for } x \in \Omega .
$$

Then for any $k \geq 1$,

$$
\int_{\mathbf{u}^{-1}\left(V_{k}\right)}|p(x)|^{s / 2}=\int_{\mathbf{u}^{-1}\left(V_{k}\right)}|q(\mathbf{u}(x))|^{s / 2} d x=\int_{V_{k}}|q(z)|^{s / 2} d z<\infty,
$$

and hence $p \in L_{\text {loc }}^{s / 2}(\Omega)$ and the pair $(\mathbf{u}, p)$ satisfies

$$
\int_{\Omega} D L(\nabla \mathbf{u}(x)): \nabla \phi(x) d x=\int_{\Omega} p(x) \operatorname{cof}(\nabla \mathbf{u}(x)): \nabla \phi(x) d x,
$$

for any $\phi \in C_{0}^{1}\left(\Omega, \mathbb{R}^{n}\right)$. In other words, $(\mathbf{u}, p)$ satisfies the system of Euler-Lagrange equations

$$
\operatorname{div}[D L(\nabla \mathbf{u}(x))-p(x) \operatorname{cof}(\nabla \mathbf{u}(x))]=0, \quad \text { in } \Omega .
$$

in the sense of (4.5). This completes the proof. 


\section{Partial Regularity of AREA-PREServing Minimizers}

For $n=2$, as a consequence of the Euler-Lagrange equations (1.7), together with the standard elliptic estimates [GM 79], we establish the following theorem.

Theorem 5.1. Let $\Omega \subset \mathbb{R}^{2}$ be a smooth, bounded simply connected domain and let $L: \mathbb{M}^{2 \times 2} \rightarrow \mathbb{R}$ be smooth, uniformly convex, such that $D L$ has linear growth and $D^{2} L$ is bounded. Let $\boldsymbol{u} \in W^{1,3}\left(\Omega, \mathbb{R}^{2}\right)$ be an areapreserving minimizer of the energy $E[\cdot]$. Furthermore, assume that the associated hydrostatic pressure $q$ on the deformed domain $\boldsymbol{u}(\Omega)$ is $C^{0, a}$ for some $0<\alpha<1$. Then $\nabla \boldsymbol{u}$ are Hölder continuous on a dense open set $\Omega_{0} \subset \Omega$.

Proof. Since $\mathbf{u} \in W^{1,3}\left(\Omega, \mathbb{R}^{2}\right)$ and $\mathbf{u}$ is area-preserving, $\mathbf{u}(\Omega)$ is open and $\mathbf{u}$ is a homeomorphism from $\Omega$ to $\mathbf{u}(\Omega)$. By Theorem 4.1, there exists $q \in L_{\text {loc }}^{3 / 2}(\mathbf{u}(\Omega))$ and the pair $(\mathbf{u}, q \circ \mathbf{u})$ satisfies the system

$$
\sum_{j=1}^{2} \frac{\partial}{\partial x_{j}}\left(\frac{\partial L}{\partial p_{j}^{i}}(\nabla \mathbf{u})-p(x)(\operatorname{cof} \nabla \mathbf{u})_{j}^{i}\right)=0, \quad \text { in } \Omega, \quad i=1,2,
$$

where $p:=q \circ \mathbf{u}$. Assume that $q \in C^{0, \alpha}(\mathbf{u}(\Omega))$. Since $\mathbf{u} \in W^{1,3}$, Sobolev imbedding theorem yields $\mathbf{u} \in C^{1 / 3}$, and hence $p(x)=q(\mathbf{u}(x))$ is Hölder continuous with the exponent $\alpha / 3$. Let $F: \Omega \times \mathbb{M}^{2 \times 2} \rightarrow \mathbb{R}$ be the free-energy defined as

$$
F(x, P):=L(P)-p(x) \operatorname{det} P \quad x \in \Omega, P \in \mathbb{M}^{2 \times 2},
$$

so that we can rewrite the nonlinear system (5.1) as

$$
\sum_{j=1}^{2} \frac{\partial}{\partial x_{j}}\left(A_{j}^{i}(x, \nabla \mathbf{u})\right)=0, \quad \text { in } \Omega, \quad i=1,2,
$$

where

$$
A_{j}^{i}(x, P):=\frac{\partial F}{\partial p_{j}^{i}}(x, P)=\frac{\partial L}{\partial p_{j}^{i}}(P)-p(x)(\operatorname{cof} P)_{j}^{i} .
$$

Let $U \subset \subset \Omega$. Since $|\operatorname{cof} P|=|P|$ for any $P \in \mathbb{M}^{2 \times 2},|D L(P)| \leq C(1+|P|)$ and $D^{2} L(P)$ is bounded,

$$
\left|A_{j}^{i}(x, P)\right| \leq C(1+|P|),\left|\frac{\partial A_{j}^{i}}{\partial p_{l}^{k}}(x, P)\right| \leq C,
$$


for any $x \in U, P \in \mathbb{M}^{2 \times 2}$. By Hölder continuity of $p$, it follows that

$$
\begin{aligned}
\frac{\left|A_{j}^{i}(x, P)-A_{j}^{i}(y, P)\right|}{1+|P|} & =|p(x)-p(y)| \frac{\left|(\operatorname{cof} P)_{j}^{i}\right|}{1+|P|} \\
& \leq C|x-y|^{\alpha / 3},
\end{aligned}
$$

for any $x \in U, P \in \mathbb{M}^{2 \times 2}$. By direct calculations and the ellipticity of $L$ it follows that

$$
\begin{aligned}
\frac{\partial A_{j}^{i}}{\partial p_{l}^{k}}(x, P) \xi_{i j} \xi_{k l} & =\frac{\partial^{2} F}{\partial p_{j}^{i} p_{l}^{k}}(x, P) \xi_{i j} \xi_{k l} \\
& =\frac{\partial^{2} L}{\partial p_{j}^{i} p_{l}^{k}}(P) \xi_{i j} \xi_{k l}-2 p(x) \operatorname{det} \xi \\
& \geq \lambda_{0}|\xi|^{2}-2 p(x) \operatorname{det} \xi \\
& :=I(x, \xi), \quad \text { for } P=\left(p_{j}^{i}\right), \xi=\left(\xi_{i j}\right) \in \mathbb{M}^{2 \times 2}
\end{aligned}
$$

where $\lambda_{0}>0$ is the ellipticity constant of $L$. Completing squares, observe that

$$
\begin{aligned}
\frac{I(x, \xi)}{\lambda_{0}}= & |\xi|^{2}-2 \frac{p(x)}{\lambda_{0}} \operatorname{det} \xi \\
= & \xi_{11}^{2}+\xi_{12}^{2}+\xi_{21}^{2}+\xi_{22}^{2}-2 \frac{p}{\lambda_{0}}\left(\xi_{11} \xi_{22}-\xi_{12} \xi_{21}\right) \\
= & \left(\xi_{11}-\frac{p}{\lambda_{0}} \xi_{22}\right)^{2}+\left(\xi_{12}-\frac{p}{\lambda_{0}} \xi_{21}\right)^{2} \\
& +\left(1-\frac{p^{2}}{\lambda_{0}^{2}}\right)\left(\xi_{22}^{2}+\xi_{21}^{2}\right) .
\end{aligned}
$$

Similarly, we obtain

$$
\frac{I(x, \xi)}{\lambda_{0}}=\left(\xi_{22}-\frac{p}{\lambda_{0}} \xi_{11}\right)^{2}+\left(\xi_{21}-\frac{p}{\lambda_{0}} \xi_{12}\right)^{2}+\left(1-\frac{p^{2}}{\lambda_{0}^{2}}\right)\left(\xi_{11}^{2}+\xi_{12}^{2}\right)
$$

Adding the identities (5.6) and (5.7), we obtain

$$
\begin{aligned}
2 \frac{I}{\lambda_{0}}= & \left(\xi_{11}-\frac{p}{\lambda_{0}} \xi_{22}\right)^{2}+\left(\xi_{12}-\frac{p}{\lambda_{0}} \xi_{21}\right)^{2} \\
& +\left(\xi_{22}-\frac{p}{\lambda_{0}} \xi_{11}\right)^{2}+\left(\xi_{21}-\frac{p}{\lambda_{0}} \xi_{12}\right)^{2}+\left(1-\frac{p^{2}}{\lambda_{0}^{2}}\right)|\xi|^{2} \\
\geq & \left(1-\frac{p^{2}}{\lambda_{0}^{2}}\right)|\xi|^{2}
\end{aligned}
$$


Thus from (5.6) and (5.8), it follows that the map $P \mapsto A(\cdot, P)$ is strongly elliptic if there exists $\mu_{0}>0$ such that

$$
\frac{\partial L_{j}^{i}}{\partial p_{l}^{k}}(x, P) \xi_{i j} \xi_{k l} \geq \frac{\lambda_{0}}{2}\left(1-\frac{p^{2}}{\lambda_{0}^{2}}\right)|\xi|^{2} \geq \mu_{0}|\xi|^{2}, \quad \text { for } x \in \Omega, P, \xi \in \mathbb{M}^{2 \times 2},
$$

which is equivalent to assume that

$$
p^{2} \leq \lambda_{0}^{2}-2 \lambda_{0} \mu_{0} \Longrightarrow\left(p-\mu_{0}\right)^{2} \leq\left(\lambda_{0}-\mu_{0}\right)^{2} .
$$

Since $p$ is defined up to addition of arbitrary constant, thus the inequality (5.9) is satisfied in subdomain $U \subset \subset \Omega$ if and only if

$$
\operatorname{osc}_{U} p<\lambda_{0}
$$

Since $p$ is Hölder continuous, the estimate (5.10) holds for any subdomain $U \subset \Omega$ with sufficiently small diameter. Hence $A(x, P)$ is strongly elliptic in $P$ for each $x \in U \subset \subset \Omega$, for sufficiently small diameter. This proves that $A_{j}^{i}(x, P)$ satisfies all the conditions of Giaquinta-Modica in GM 79 on $U \subset \subset \Omega$, with diameter of $U$ being small. Hence by GM 79, Theorem 1], we conclude that $\nabla \mathbf{u}$ is Hölder continuous on a dense open subset $U_{0}$ of $U$. By standard covering arguments we conclude the proof.

Acknowledgement This work was initiated while both the authors were at the Australian National University, which was supported by Australian Research Council. The second author was partially supported by the National Science Foundation.

\section{REFERENCES}

[Ba 77] Ball,J.: Convexity conditions and existence theorems in nonlinear elasticity. Arch. Rat. Mech. Anal. 64, 337-403 (1977).

[BOP 92] Bauman,P., Owen,N.C., Phillips,D.: Maximum principles and an a priori estimates for an incompressible material in nonlinear elasticity. Comm. Partial Differential Equations 17, 1185-1212 (1992).

[CZ 52] Calderón,A.P., Zygmund,A.: On the existence of certain singular integrals. Acta Math. 88, 85-139 (1952).

[CKS 93] Chang, D.C; Krantz, S.G.; Stein, E.M.: $H^{p}$ theory on a smooth domain in $\mathbb{R}^{N}$ and elliptic boundary value problems. J. Funct. Anal. 114 286-347 (1993).

[DL 88] R. Dautry, J. L. Lions, Mathematical Analysis and Numerical Methods for Science and Technology, Volume 2 Functional and Variational Methods, Springer (1988).

[Ev 98] Evans,L.C.: Partial Differential Equations. Graduate Studies in Mathematics, 19, American Mathematical Society, 1998.

[EG 99] Evans,L.C., Gariepy,R.F,: On the partial regularity of energy-minimizing, area-preserving maps, Calc. Var. Partial Differential Equations 9, 357-372 (1999). 
[Fe 71] Fefferman, C.: Characterizations of bounded mean oscillation. Bull. Amer. Math. Soc. 77, 587-588 (1971).

[FS 72] Fefferman, C,; Stein, E.M.: $H^{p}$ spaces of several variables. Acta Mathematica, 129, 137-193 (1972)

[GM 79] Giaguinta,M., Modica,G.: Almost-everywhere regularity results for solutions of nonlinear elliptic systems. Manuscripta Math. 28, 109-158 (1979).

[Go 79] Goldberg, D.: A local version of real Hardy spaces. Duke Math. J. 46, 27-42 (1979).

[GT 97] Gilbarg,D. Trudinger,N.S.: Elliptic partial differential equations of second order. Reprint of the 1998 edition. Classics in Mathematics, SpringerVerlag, Berlin, 2001.

[TO 81] LeTallec,P., Oden,J.T.: Existence and characterization of hydrostatic pressure in finite deformations of incompressible elastic bodies. J. Elasticity 11, 341-357 (1981).

[Mi 90] $\quad H^{p}$ spaces over open subsets of $\mathbb{R}^{n}$. Studia Math. 95, 205-228 (1990).

[Mo 52] Morrey,C.B.: Quasiconvexity and the semicontinuity of multiple integrals. Pacific. J. Math. 2, 25-52 (1952).

[Og 84] Ogden,R.W.: Non-linear elastic deformations. Ellis Horwood Ltd. Chichester, (1984).

[St 70] Stein,E.: Singular integrals and differentiability properties of functions. Princeton University Press, Princeton, NJ, (1970).

[St 93] Stein, E. M. Harmonic analysis: Real-variable methods, Orthogonality, and Oscillatory integrals. Princeton Mathematical Series; 43, Princeton University Press, Princeton, NJ, (1993).

[Sv 88] Šverák,V.: Regularity properties of deformations with finite energy. Arch. Rat. Mech. Anal. 100, 105-127 (1988).

[Te 01] R. Temam: Navier-Stokes Equations: Theory and Numerical Analysis. AMS Chelsha Publishing, AMS, Rhode Island, (2001).

School of Mathematics and Applied Statistics University of WollonGOnG, Wollongong, NSW 2522, Australia

E-mail address: chaudhur@uow.edu.au

Department of Mathematics, University of Texas at Austin,Austin, TEXAS 78712, USA

E-mail address: aram@math.utexas.edu 